\title{
Prevalência de glaucoma identificada em campanha de detecção em São Paulo
}

\author{
Prevalence of glaucoma in a screening program in São Paulo
}

\author{
Cristine Araújo Póvoa ${ }^{1}$ \\ Marcelo Teixeira Nicolela ${ }^{2}$ \\ Ana Letícia Siqueira Leão Valle ${ }^{3}$ \\ Luís Eduardo de Siqueira Gomes ${ }^{4}$ \\ Isaac Neustein ${ }^{5}$
}

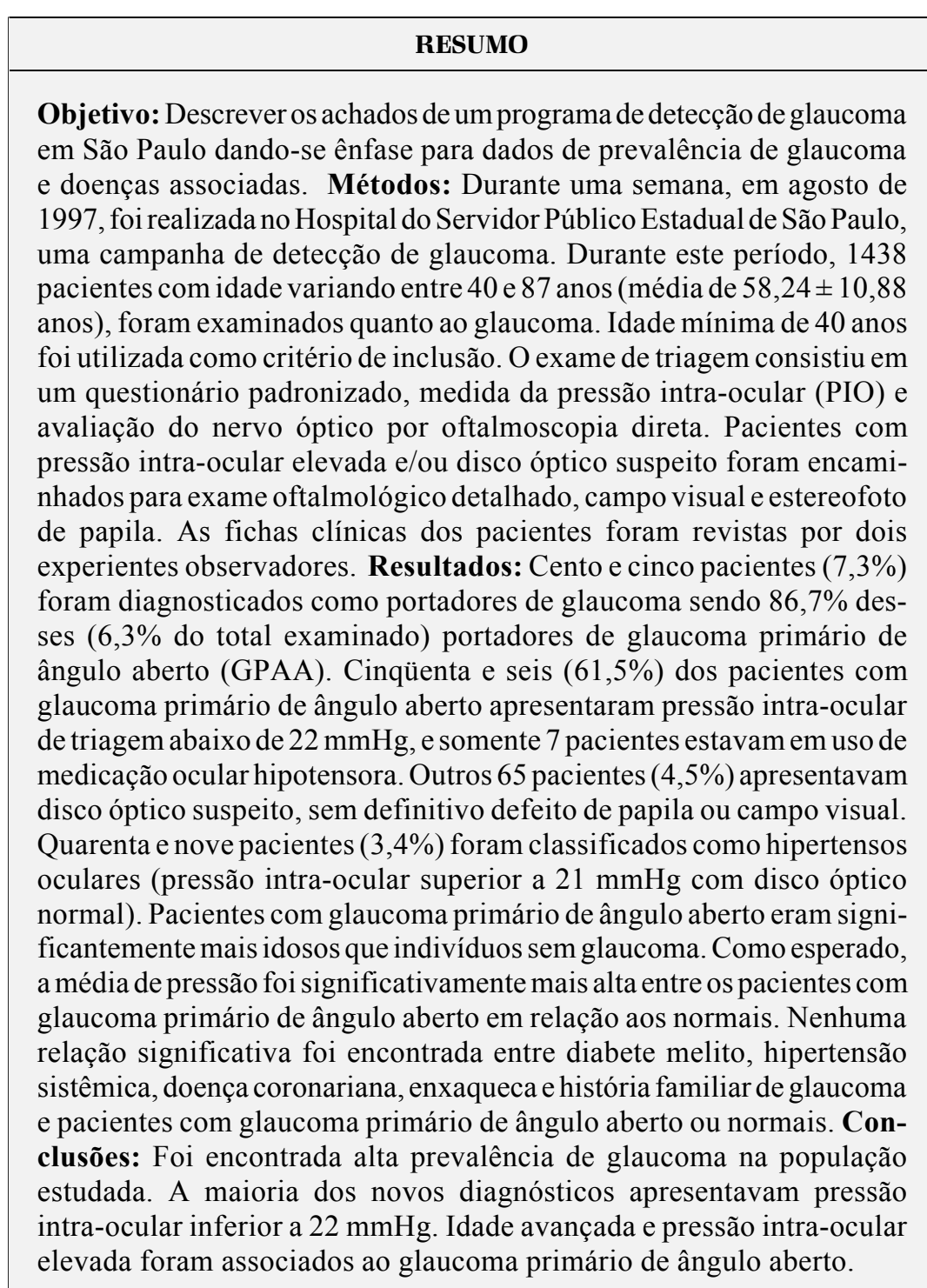

Descritores: Glaucoma de ângulo aberto/diagnóstico; Glaucoma de ângulo aberto/ epidemiologia; Hipertensão ocular/fisiopatologia; Promoção da saúde; Saúde ocular

\section{INTRODUÇ̃̃̃O}

O glaucoma é uma doença que cursa com lesão progressiva do nervo óptico e perda característica do campo visual, podendo levar à cegueira. Estima-se que, no ano 2000, cerca de 66,8 milhões de pessoas sejam porta- 
doras de glaucoma e, dessas, 6,7 milhões sejam bilateralmente $\operatorname{cegas}^{(1)}$.

Em decorrência da evolução insidiosa e assintomática do glaucoma nos estágios iniciais, o seu diagnóstico é geralmente realizado tardiamente. Para diagnóstico precoce da doença é fundamental a conscientização da população e dos profissionais de saúde envolvidos no atendimento oftalmológico. Mesmo nos países desenvolvidos, estudos populacionais mostram que aproximadamente metade dos pacientes identificados com glaucoma desconhecia ser portador da doença $\mathrm{a}^{(2-3)}$. Considerando as condições precárias de assistência à saúde nas nações em desenvolvimento, essa situação pode ser pior em países como o Brasil.

Estima-se que a prevalência de glaucoma primário de ângulo aberto (GPAA) em povos de origem européia seja de $2,42 \%$ $(\mathrm{DP} \pm 2,10 \%)$ em pacientes acima de 40 anos, tendo associação positiva e exponencial com a idade. Nos povos de origem africana a prevalência de glaucoma é muito maior, e apresenta relação linear com a idade ${ }^{(1)}$. Nos países da América Latina, incluindo o Brasil, as estimativas sobre prevalência de glaucoma não são totalmente fidedignas, principalmente pela dificuldade de submeter-se uma amostra populacional significativa a todos os exames necessários para o diagnóstico da doença ${ }^{(4-5)}$.

Este estudo tem a finalidade de descrever os achados de um programa de detecção de glaucoma em São Paulo, dandose ênfase para os dados de prevalência de glaucoma e de doenças associadas na população estudada.

\section{MÉTODOS}

De 27 a 31 de agosto de 1997 realizou-se no Hospital do Servidor Público do Estado de São Paulo (HSPE-SP) uma campanha de detecção de glaucoma na população geral. O protocolo do estudo foi avaliado e aprovado pelo Comitê de Ética do Hospital. Utilizou-se como critério de inclusão idade igual ou superior a 40 anos.

Os indivíduos examinados tomaram conhecimento da campanha através dos meios de comunicação (rádio, televisão, jornais), e compareceram voluntariamente para exame.

O exame oftalmológico foi dividido em duas etapas: uma de triagem e outra de confirmação do diagnóstico dos pacientes identificados como suspeitos.

$\mathrm{Na}$ fase de triagem cada indivíduo preencheu um questionário fornecendo dados de identificação (nome, idade, sexo, cor); dados quanto aos antecedentes patológicos (história prévia de diabetes melito, hipertensão arterial sistêmica, doença coronariana, enxaqueca, glaucoma); dados quanto aos antecedentes familiares de glaucoma. Não foram realizados exames comprobatórios das doenças investigadas.

A seguir, realizou-se medida da pressão intra-ocular (PIO) com pneumotonômetro das marcas Nidek NT 2000 e Reichert e fundoscopia direta. Caso o valor medido da PIO fosse maior ou igual a $21 \mathrm{mmHg}$ em um dos olhos, era novamente aferida em tonômetro de aplanação de Goldmann.
Todos os pacientes, avaliados na fase de triagem, cuja PIO medida em tonômetro de Goldmann apresentasse valor maior ou igual a $21 \mathrm{mmHg}$ e/ou disco óptico com pelo menos uma das seguintes características à fudoscopia direta, foram encaminhados para a segunda fase do exame:

- relação escavação sobre disco (E/D) igual ou superior a 0,5;

- e/ou assimetria de disco igual ou superior a 0,2 ;

- e/ou presença de afinamento de rima localizada;

- e/ou hemorragia de papila;

- e/ou palidez localizada ou difusa.

Nessa fase, os pacientes suspeitos foram submetidos ao exame oftalmológico completo (incluindo gonioscopia e biomicroscopia de papila), e mantendo-se a suspeita de glaucoma, realizou-se exame de campo visual automatizado e estereofotografia de papila. A campimetria foi realizada com perímetro Haag-Streit Octopus 1-2-3, programa 1Gx (Interzeag AG, CH 8952 Schlieren), ou com perímetro Humphrey Field Analyser II (San Diego-California), programa C 24-2. A estereofotografia de papila foi realizada com retinógrafo marca Zeiss Germany.

Ao final da avaliação, os pacientes foram orientados quanto à doença e seu controle. Caso houvesse sido diagnosticado glaucoma, a medicação inicial foi fornecida e o paciente referido para acompanhamento. Suspeitos de glaucoma e hipertensos oculares foram também encaminhados para acompanhamento. Indivíduos normais foram liberados com orientação.

As fichas clínicas de todos os indivíduos atendidos na campanha, campos visuais e estereofotos de papila foram posteriormente revistas por dois examinadores experientes. A avaliação das estereofotos do disco óptico foi realizada de forma simultânea pelos dois observadores, obtendo-se consenso entre eles quanto à presença de dano glaucomatoso.

Os pacientes foram então classificados em um dos quatro grupos definidos abaixo, de acordo com os seguintes critérios:

- Normais: indivíduos com PIO normal e disco óptico sem características de glaucoma;

- Hipertensos oculares: pacientes com PIO maior ou igual a $21 \mathrm{mmHg}$ com disco óptico normal e com campo visual sem alterações compatíveis com glaucoma, segundo critérios definidos abaixo;

- Suspeitos de glaucoma: pacientes com disco óptico suspeito de apresentar dano glaucomatoso (relação $\mathrm{E} / \mathrm{D} \geq$ a 0,7 ou assimetria de escavação $>$ que 0,2 sem outras alterações de anel neuroretiniano sugestivas de glaucoma), porém com campo visual normal, independentemente dos níveis de PIO;

- Glaucoma: pacientes com disco óptico francamente glaucomatoso, com ou sem defeito de campo visual, independente dos níveis de PIO ou paciente com disco óptico inicialmente suspeito cujo defeito se confirmou por alteração campimétrica compatível;

O disco óptico foi considerado francamente glaucomatoso quando apresentava:

- relação $\mathrm{E} / \mathrm{D} \geq \mathrm{a}$ 0,8, com rima neural não homogênea; 


\begin{tabular}{|c|c|c|c|c|c|c|}
\hline & & $\begin{array}{l}\text { Normais } \\
n=1219\end{array}$ & $\begin{array}{l}\text { Hipertensos Oculares } \\
\qquad n=49\end{array}$ & $\begin{array}{l}\text { Suspeitos de Glaucoma } \\
\qquad n=65\end{array}$ & $\begin{array}{c}\text { Glaucoma } \\
n=105\end{array}$ & valor de $p$ \\
\hline \multicolumn{7}{|l|}{ Gênero } \\
\hline Masculino & n (\%) & $358(29,4)$ & $15(30,6)$ & $23(35,4)$ & $46(43,8)$ & 0,11 \\
\hline Feminino & $\mathrm{n}(\%)$ & $861(70,6)$ & $34(69,4)$ & $42(64,6)$ & $59(56,2)$ & \\
\hline \multicolumn{7}{|l|}{ Raça * } \\
\hline Branca & n (\%) & $869(73,0)$ & $38(80,9)$ & $39(60,9)$ & $76(74,5)$ & 0,02 \\
\hline Negra & n (\%) & $53(4,5)$ & $3(6,4)$ & $9(14,1)^{*}$ & $3(2,9)$ & \\
\hline Parda & n (\%) & $32(2,7)$ & $1(2,1)$ & $1(1,6)$ & $5(4,9)$ & \\
\hline Amarela & n (\%) & $237(19,9)$ & $5(10,6)$ & $15(23,4)$ & $18(17,6)$ & \\
\hline \multicolumn{7}{|l|}{ Idade } \\
\hline Média \pm DP & & $57,6 \pm 18,8$ & $59,9 \pm 9,7$ & $57,6 \pm 11,0$ & $63,6 \pm 11,9$ & 0,008 \\
\hline
\end{tabular}

- relação E/D > 0,5 com ausência completa de tecido nervoso estendendo-se até a borda do disco (notch).

O campo visual foi considerado anormal de acordo com o seguinte critério: presença de pelo menos três pontos do mesmo hemicampo, agrupados, com "pattern deviation" alterado $(\mathrm{p} \leq 2 \%)$, sendo que pelo menos um dos pontos apresentava $p \leq 1 \%$. Esses pontos alterados deveriam estar localizados em áreas compatíveis com defeito no feixe de fibras nervosas.

Os dados obtidos foram analisados estatisticamente utilizando o programa informatizado SPSS (SPSS Inc., Chicago, IL). A análise entre grupos foi realizada por meio dos testes do Qui-quadrado $\left(\mathrm{X}^{2}\right)$ para variáveis nominais e análise de variância com correção para comparações múltiplas de Bonferroni para variáveis contínuas. Valores de $p<0,05$ foram considerados significantes.

\section{RESULTADOS}

Durante a campanha de detecção de glaucoma, 1438 indivíduos com idade acima de 40 anos foram examinados. Desses, 442 (30,74\%) eram do sexo masculino e $996(69,26 \%)$ do sexo feminino. A idade variou entre 40 e 87 anos, com média de 58,24 anos ( $\mathrm{DP} \pm 10,88$ anos). Os demais dados demográficos de acordo com os 4 grupos diagnósticos, estão dispostos na tabela 1. Pode-se observar que houve diferença significante na distribuição dos pacientes quanto à raça nos 4 grupos, com prevalência significantemente maior de indivíduos da raça negra entre os suspeitos de glaucoma. Também no que diz respeito à idade média, pacientes com glaucoma eram significantemente mais velhos que indivíduos normais e pacientes suspeitos de glaucoma.

Foram diagnosticados 105 pacientes $(7,3 \%)$ como portadores de glaucoma, sendo que 92 desses $(87,6 \%$ dos diagnosticados como portadores de glaucoma ou $6,4 \%$ do total examinado) não tinham diagnóstico prévio da doença. Somente 13 pacientes portadores de glaucoma $(12,4 \%$ dentre os diagnosticados) estavam cientes de sua patologia. Dos pacientes com glaucoma, 91 (6,3\%) eram portadores de glaucoma primário de ângulo aberto (GPPA) sendo que 56 (3,9\%) apresentavam PIO de triagem abaixo de $22 \mathrm{mmHg}$ e somente 7 estavam em uso de medicação hipotensiva. Oito $(0,6 \%)$ pacientes apresentavam glaucoma de ângulo fechado e $6(0,4 \%)$ glaucoma secundário. Outros 65 pacientes $(4,5 \%)$ foram classificados como suspeitos de glaucoma e 49 pacientes $(3,4 \%)$ foram classificados como hipertensos oculares. A prevalência dos grupos diagnosticados encontra-se na tabela 2.

Em relação à prevalência de doenças associadas, 161 $(11,4 \%)$ relatavam diabetes melito $(\mathrm{DM})$ e $538(38,0 \%)$ relatavam hipertensão arterial sistêmica (HAS). A prevalência dessas e outras patologias nos pacientes com ou sem glaucoma pode ser verificado na tabela 3 . Não houve diferença significante na prevalência de DM, HAS, enxaqueca, doença coronariana e história familiar entre pacientes com ou sem glaucoma.

Como se esperava, a média da PIO foi significantemente maior nos pacientes com GPAA em relação aos indivíduos normais $(19,9 \pm 6,9$ versus $15,0 \pm 3,4 \mathrm{mmHg}$ no olho direito e $19,6 \pm 5,2$ versus $14,9 \pm 3,3 \mathrm{mmHg}$ no olho esquerdo, respectivamente $-\mathrm{p}<0,001)$.

\section{DISCUSSÃO}

A prevalência e o tipo de glaucoma variam consideravelmente de acordo com a população estudada. Diversos estudos relatam uma maior prevalência de GPAA em pacientes da raça negra, que também apresentam doença mais grave ${ }^{(3)}$. Por

\begin{tabular}{|lcc|}
\hline \multicolumn{3}{|c|}{ Tabela 2. Prevalência dos pacientes nos grupos diagnosticados } \\
Grupos Identificados & Freqüência & Prevalência \\
Normais & 1206 & $83,9 \%$ \\
Suspeitos & 65 & $4,5 \%$ \\
Hipertensos oculares & 49 & $3,4 \%$ \\
Glaucoma crônico simples & & \\
PIO de triagem $>21$ & 35 & $2,4 \%$ \\
PIO de triagem $<21$ & 56 & $3,9 \%$ \\
Glaucoma de ângulo fechado & 08 & $0,6 \%$ \\
Glaucoma secundário & 06 & $0,4 \%$ \\
\hline
\end{tabular}


Tabela 3. Prevalência de pacientes com Diabetes melito, Hipertensão arterial sistêmica, Doença coronariana, Enxaqueca e História familiar de glaucoma

\begin{tabular}{|c|c|c|c|c|c|}
\hline Fatores de risco & $\begin{array}{l}\text { Pacientes com glaucoma } \\
\qquad(\%)\end{array}$ & $\begin{array}{c}\text { Normais } \\
\mathrm{N}(\%)\end{array}$ & $\begin{array}{c}\text { Suspeitos de glaucoma } \\
\mathrm{N}(\%)\end{array}$ & $\begin{array}{c}\text { Hipertensos oculares } \\
\mathrm{N}(\%)\end{array}$ & $\begin{array}{l}\text { Total } \\
\mathrm{N}(\%)\end{array}$ \\
\hline DM & $15(14,3)$ & $135(11,3)$ & $4 \quad(6,2)$ & $7 \quad(14,3)$ & $161 \quad(11,4)$ \\
\hline HAS & $49(46,7)$ & $445(37,2)$ & $23(35,4)$ & $21(42,9)$ & $538 \quad(38,0)$ \\
\hline Doença coronariana & $11(10,5)$ & $128(10,9)$ & $9 \quad(14,5)$ & $2 \quad(4,1)$ & $150 \quad(10,8)$ \\
\hline Enxaqueca & $25(23,8)$ & $322(26,9)$ & $15(23,4)$ & $10(20,4)$ & $372 \quad(26,3)$ \\
\hline História Familiar & $21(20,0)$ & $218(18,7)$ & $21(32,8)$ & $13(27,1)$ & $273(19,78)$ \\
\hline
\end{tabular}

* 24 pacientes não informaram sobre DM; 22 sobre HAS; 45 sobre doença coronariana; 23 sobre enxaqueca e 58 sobre história familiar de glaucoma. DM: Diabetes melito; HAS: Hipertensão arterial sistêmica.

outro lado, o glaucoma de ângulo fechado é muito mais prevalente em determinadas populações, como por exemplo chineses e esquimós ${ }^{(6)}$. Já em escandinavos, o glaucoma pseudoesfoliativo supera o GPAA em termos de prevalência em indivíduos idoso $^{(7)}$.

Estudos populacionais realizados nos EUA e em países da Europa, revelaram uma prevalência de glaucoma de aproximadamente 2,0\%, em pacientes acima de 40 anos $^{(1)}$. Em nosso estudo, encontramos 7,3\% de pacientes afetados. Esta diferença decorre, muito provavelmente, de não se tratar de um estudo populacional, mas sim de uma campanha de detecção da doença, com uma população que procurou de forma espontânea nosso Serviço durante a campanha. Supõe-se, portanto, que pacientes com mais fatores de risco conhecidos viriam mais prontamente ao exame e pacientes com diagnóstico suspeito ou confirmado de glaucoma estariam mais predispostos a comparecer e avaliar o estado de sua doença, o que poderia explicar esta tendenciosidade. Contudo, do total de indivíduos examinados durante a campanha, somente 20 pacientes $(1,4 \%)$ reportaram ter glaucoma, embora o diagnóstico não tenha sido confirmado em 7 (35\%) desses casos.

Ghanem, em campanha realizada em Joinville, encontrou uma prevalência de $1,75 \%$ na população estudada acima de 40 anos. Entretanto, esse estudo abordou uma população com forte descendência européia e pouca miscigenação racial se comparada com a população de São Paulo. Além disso, não foram realizadas biomicroscopia do disco óptico ou estereofoto, o que pode subestimar algumas alterações no disco. Dos pacientes portadores de glaucoma, somente 7,29\% sabiam ser portadores da doença, sendo que apenas 3,12\% estavam em uso de medicação ${ }^{(4)}$.

Em estudo da morbidade ocular em idosos (65 anos ou mais) realizado pela Secretaria da Saúde do Estado de São Paulo, em três subdistritos da cidade, o glaucoma foi a quarta principal causa de deficiência visual ${ }^{(5)}$.

Não se pode descartar, entretanto, a possibilidade de que a prevalência de glaucoma na nossa população seja maior do que a de países desenvolvidos do hemisfério norte e deve-se salientar, aqui, a necessidade de realização de estudos populacionais sobre a prevalência e impacto social do glaucoma no Brasil.
Outro dado relevante obtido no estudo foi de que 56 dos 91 pacientes $(61,5 \%)$ dos portadores de GPAA apresentaram PIO de triagem menor que $22 \mathrm{mmHg}$. Esses valores estão de acordo com os encontrados em diversos estudos populacionais ${ }^{(3,8)}$ nos quais a PIO de triagem de aproximadamente metade dos pacientes diagnosticados com glaucoma, encontrava-se dentro da normalidade. Uma diferença, entretanto, é que nesses estudos cerca de metade dos pacientes identificados como glaucomatosos, já se encontravam utilizando medicação hipotensora, o que não ocorreu em nosso estudo, no qual apenas $12,4 \%$ dos pacientes utilizavam medicação.

A correlação entre a hipertensão arterial e diabetes em pacientes com glaucoma é discutível. Mostra-se elevada principalmente nos estudos realizados em clínicas oftalmológicas nas quais a freqüência de pacientes com diabetes e hipertensão provavelmente também é elevada pela própria tendência destes pacientes serem triados para esses centros ${ }^{(9-14)}$. Estudos populacionais encontraram associação fraca ou não associação entre DM, HAS e glaucoma ${ }^{(8,15)}$, com exceção do estudo de Rotterdam ${ }^{(16)}$. Em nosso estudo não foi encontrada correlação entre essas afecções. Deve-se ressaltar, entretanto, que a presença ou não dessas doenças foi relatada pelos pacientes, e não foram realizados exames que comprovassem a informação obtida.

A associação de enxaqueca e glaucoma, particularmente do glaucoma de pressão normal foi relatada inicialmente por Phelps e Corbett ${ }^{(17)}$. Posteriormente diversos autores confirmaram uma prevalência aumentada de enxaqueca em pacientes com glaucoma e outros distúrbios supostamente vasoespásticos ${ }^{(10,18)}$. Essa associação é contestada por outros autores ${ }^{(19)}$. Neste estudo não observamos diferença significante na prevalência de enxaqueca em indivíduos normais e glaucomatosos. As mesmas ressalvas feitas em relação aos dados obtidos quanto ao DM e HAS devem ser estendidas à enxaqueca: não foi realizado nenhum exame comprobatório da existência ou não de enxaqueca, sendo a informação fornecida pelo paciente.

A PIO é o principal fator de risco para o glaucoma. Vários estudos populacionais demonstram a alta prevalência de glaucoma relacionada ao aumento da $\mathrm{PIO}^{(3)}$. Encontramos PIO significantemente elevada nos portadores de GPAA em comparação aos normais. 


\section{CONCLUSÃO}

Foi detectada uma alta prevalência de glaucoma na população estudada sendo que a maior parte dos pacientes glaucomatosos apresentavam PIO normal na medida de triagem. Considerando a importância do glaucoma como segunda causa de cegueira no mundo é imprescindível a realização de estudos que caracterizem adequadamente o glaucoma em nossa população e a oriente quanto a prevenção. Não houve associação entre DM, HAS, enxaqueca e glaucoma nesta população.

\section{ABSTRACT}

Purpose: To evaluate the prevalence of glaucoma and associated risk factors diagnosed during a glaucoma screening program. Methods: A screening program for detection of glaucoma was carried out in a major public hospital in São Paulo for one week in August 1997. During this period, 1438 patients with age between 40 and 87 years (average 58.24 10.88) were evaluated regarding glaucoma. The inclusion criterion was age of at least 40 years. The screening examination consisted of a standardized questionnaire, intraocular pressure (IOP) and optic nerve assessment by direct ophthalmoscopy. Patients with: increased intraocular pressure and / or suspicious optic discs were referred to further tests (complete ocular examination, automated visual field test and optic disc stereophotograph). The clinical chart of all patients were reviewed by two experienced observers, using predefined criteria. Results: A hundred and five patients (7.3\%) were identified having glaucoma, 91 of these $(86.7 \%)$ with primary open angle glaucoma (POAG). Fifty six of the POAG patients $(61.5 \%)$ had screening IOP below $22 \mathrm{mmHg}$, and only 7 of these were using ocular hypotensive medications. Other 65 patients $(4.5 \%)$ had suspicious optic disc appearance, without definitive disc or field damage. 49 patients (3.4\%) were classified as ocular hypertensives (intraocular pressure over $21 \mathrm{mmHg}$ with normal disc). Patients with POAG were significantly older than subjects without glaucoma. As expected, the mean IOP was significantly higher in POAG patients than in normal. No significant difference was observed between subjects with diabetes, systemic hypertension, coronary disease, migraine and family history of glaucoma and POAG patients and normals. Conclusions: A high prevalence of glaucoma was observed during this screening program. The majority of newly diagnosed cases had intraocular pressure below $22 \mathrm{mmHg}$.
Older age and elevated intraocular pressure was associated with the presence of primary open angle glaucoma.

Keywords: Open-angle glaucoma/diagnosis; Open-angle glaucoma/epidemiology; Ocular hypertension/physiopathology; Health promotion; Eye health.

\section{REFERÊNCIAS}

1. Quigley HA. Number of people with glaucoma worldwide. [commented on $\mathrm{Br}$ J Ophthalmol 1996;80:385-7]. Br J Ophthalmol 1996;80:389-93.

2. Sheldrick JH, Sharp AJ. Glaucoma screening clinic in general practice: prevalence of occult disease, and resource implications. [commented on $\mathrm{Br} \mathrm{J}$ Gen Pract 1995;45:383]. Br J Gen Pract 1994;44:561-5.

3. Sommer A, Tielsch JM, Katz J, Quigley HA, Gottsch JD, Javitt J, Singh K. Relationship between intraocular pressure and primary open angle glaucoma among white and black americans. The Baltimore Eye Survey. Arch Ophthalmol 1991;109:1090-5.

4. Ghanem CC. Levantamento de casos de Glaucoma em Joinville - Santa Catarina, 1984. Arq Bras Oftalmol 1989;52:40-3.

5. Medina NH, Barros OM de, Muños EH, Magdaleno RL, Barros AJA, Dornellas J de, et al. Morbidade ocular em idosos da cidade de São Paulo-SP, Brasil. Arq Bras Oftalmol 1993;56:276-83.

6. Congdon N, Wang F, Tielsch JM. Issues in the epidemiology and population-based screening of primary angle-closure glaucoma. Surv Ophthalmol 1992;36:411-23

7. Ringvold A, Blika S, Elsas T, Guldahl J, Juel E, Brevik T et al. The Middle -Norway eye screening study II. Prevalence of simple and capsular glaucoma. Acta Ophthalmol (Copenh) 1991;69:561-4.

8. Kahn HA, Leibowitz HM, Ganley JP, Kini MM, Colton T, Nickerson KS, Dawber TR. The Framingham Eye Study II. The association of ophthalmic pathology with single variables previously measured in the Framingham Heart Study. Am J Epidemiol 1977; 106:33-41.

09. Leske MC, Connell MS, Wu SY, Hyman LG, Schachat AP. Risk factors for open angle glaucoma. The Barbados Eye Study. [commented on Arch Ophthalmol 1996;114:235]. Arch Ophthalmol 1995;113:918-24.

10. Morgan RW, Drance SM. Chronic open-angle glaucoma and ocular hipertension. An epidemiological study. Br J Ophthalmol 1975;59:211-5.

11. Klein B, Klein R. Intraocular pressure and cardiovascular risk variables. Arch Ophthalmol 1991;99(Suppl 5):837-9.

12. Becker B. Diabetes Mellitus and primary open angle glaucoma. The XXVII Edward Jackson Memorial Lecture. Am J Ophthalmol 1971;1(1 Pt 1):1-16.

13. Klein BE, Klein R, Jense SC. Open-angle glaucoma and older-onset diabetes. The Beaver Dam Eye Study. Ophthalmology 1994;101:1173-7.

14. Lieb WA, Stark N, Jelinek MB, Malzi R. Diabetes mellitus and glaucoma Acta Ophthalmol (Copenh)1967;(Suppl 94):3-62.

15. Tielsch JM, Katz J, Quigley HA, Javitt JC, Sommer A. Diabetes, intraocular pressure and primary open-angle glaucoma in the Baltimore Eye Survey. Ophthalmology 1995;102:48-53.

16. Dielemans I, Vingerling JR, Algra D, Hofman A, Grobbee DE, Jong PTVM. Primary open-angle glaucoma, intraocular pressure, and systemic blood pressure in the general elderly population. The Rotterdam Study. [commented on Ophthalmology 1995;102:1126]. Ophthalmology 1995;102:54-60.

17. Phelps CD, Corbett JJ. Migraine and low tension glaucoma. A case control study. Invest Ophthalmol Vis Sci 1985;26:1105-8.

18. Wang JJ, Mitchel P, Smith W. Is there an association between migraine headache and open angle glaucoma? Findings from the Blue Montains Eye Study. Ophthalmology 1997;104:1714-9.

19. Usui T, Iwata K, Shirakashi M, Abe H. Prevalence of migraine in low tension glaucoma and primary open angle glaucoma in japanese. $\mathrm{Br} \mathrm{J}$ Ophthalmol 1991;75:224-6. 